\title{
A phase I study of tivantinib in combination with temsirolimus in patients with advanced solid tumors
}

\author{
Christos E. Kyriakopoulos ${ }^{1}$, Amy M. Braden ${ }^{1}$, Jill M. Kolesar ${ }^{1}$, Jens C. Eickhoff ${ }^{1}$, Howard H. \\ Bailey $^{1}$, Jennifer Heideman ${ }^{1}$, Glenn Liu ${ }^{1}$, and Kari B. Wisinski ${ }^{1}$ \\ ${ }^{1}$ University of Wisconsin Carbone Cancer Center, University of Wisconsin-Madison, Madison, WI
}

\section{Summary}

Background-A wide variety of human cancers exhibit dysregulated c-Met activity that has implications in oncogenesis. Phosphorylation of c-Met results in activation of the PI3K/AKT/ mTOR pathway. Combined blockade of c-Met and mTOR pathways has shown efficacy in preclinical studies. Tivantinib is a c-Met inhibitor and temsirolimus is a selective mTOR inhibitor. We aimed to determine the maximum tolerated dose (MTD) and the recommended phase II dose (RP2D), dose-limiting toxicities (DLT), adverse events (AEs), clinical activity and pharmacokinetic $(\mathrm{PK})$ parameters of the combination.

Methods-This open-label phase I study used a $3+3$ dose escalation design. Patients (pts) were treated with escalating doses of tivantinib (120-360 mg tablets orally twice daily) and temsirolimus (20 mg IV weekly) followed by dose expansion at the MTD. Separate cohorts were planned for extensive (normal) and poor tivantinib metabolizers based on CYP2C19 genotypes. Cycles were 28 days besides cycle 1 that was 35 days to allow for PK analysis.

Results-Twenty-nine pts [median age 58 (range 28-77)] were enrolled (21 in dose escalation and 8 in dose expansion). All were extensive CYP2C19 metabolizers. The most common types of cancer were colorectal, ovarian and non-small cell lung. Sixteen out of 21 and 6 out of 8 pts were evaluable for DLT evaluation per protocol in the dose escalation and dose expansion phases, respectively. Pts remained on study for a median of 71 days (range 18-296). The MTD and RP2D was tivantinib $240 \mathrm{mg}$ twice daily and temsirolimus $20 \mathrm{mg}$ weekly. DLTs included grade (gr) 4 neutropenia ( 2 pts; 1 with gr 3 febrile neutropenia), gr 3 abdominal pain ( 1 pt) and gr 2 mucositis resulting in inadequate drug delivery. The most common treatment related AEs grade $\geq 2$ included: anemia (gr 2 in 9 pts, gr 3 in 3 pts), fatigue (gr 2 in 10 pts), anorexia (gr 2 in 9 pts), hypoalbuminemia (gr 2 in 6 pts, gr 3 in 2 pts), hypophosphatemia (gr 2 in 2 pts, gr 3 in 5 pts) and nausea (gr 2 in 6 pts, gr 3 in 1 pt). One pt with ovarian cancer had a confirmed partial response and remained on study for 10 months, a second patient with ovarian cancer had stable disease and remained on study for 6 months and a third pt with squamous cell carcinoma of the tongue had stable disease and remained on study for 7 months. Pharmacokinetic analysis showed that there is no interaction in the plasma concentrations between tivantinib and temsirolimus.

Corresponding author: Christos E. Kyriakopoulos, MD, University of Wisconsin Carbone Cancer Center, 600 Highland Avenue, Madison, WI 53792, Phone: (608) 263-0786, Fax: (608) 265-0614, ckyriako@ medicine.wisc.edu.

Conflict of interest: The authors declare that they have no conflict of interest. 
Conclusions-The combination of tivantinib with temsirolimus appears to be well tolerated with evidence of clinical activity.

\section{Keywords}

Tivantinib; temsirolimus; Phase I; c-Met; PI3K/AKT/mTOR

\section{Introduction}

Receptor tyrosine kinases (RTKs) are cell surface receptors for growth factors, hormones and cytokines that regulate a plethora of signal transduction pathways involved in multiple normal and pathologic biological processes [1]. In normal cells RTK activity is under tight control, whereas in cancer cells they can be severely dysregulated, leading to oncogenesis, invasiveness, metastasis and resistance to treatment [2]. Thus, RTKs have emerged as an important class of molecular targets for anticancer therapy.

Dysregulated c-Met activity is exhibited in a wide variety of human cancers, either through over-expression of the c-Met kinase, activating mutations in c-Met, or increased autocrine or paracrine secretion of the c-Met ligand hepatocyte growth factor/scatter factor (HGF/SF) [3, 4]. These alterations have been strongly implicated in tumor progression and metastasis in a variety of cancers, and a high constitutive activation of the c-Met RTK has been correlated with poor clinical prognosis [3]. c-Met can be activated in both a ligand-dependent manner, by the overexpression of c-Met and/or its ligand HGF, or a ligand-independent manner as in the case of activating mutations of c-Met, such as those described in sporadic and hereditary papillary renal carcinoma [5]. Activation and autophosphorylation of c-Met results in the binding and phosphorylation of adaptor proteins such as Gab1, Grb2, Shc and c-Cbl, and results in the subsequent activation of signaling pathways, including the PI3K/AKT/mTOR, FAK, STAT and Ras/MEK/ERK pathways that play pivotal roles in cell survival, proliferation, invasion, and angiogenesis [6,7].

The presence of c-Met in a wide variety of cancers and its role in controlling multiple signal transduction pathways involved in tumor growth renders it an attractive therapeutic target for human cancer. Tivantinib (ARQ 197; ArQule, Inc., Daiichi Sankyo, Co., Ltd; Kyowa Hakko Kirin, Co., Ltd) is a selective, oral, small molecule tyrosine kinase inhibitor of c-Met RTK [8]. It is metabolized primarily by CYP2C19 and partially by CYP3A4/5 [9]. Two previous phase I studies with single agent tivantinib have shown a favorable safety profile with a recommended phase II (RP2D) dose at $360 \mathrm{mg}$ twice daily as monotherapy [10, 11], although in CYP2C19 poor metabolizers the RP2D was 240mg twice daily [12]. In phase II studies, tivantinib as single agent has shown activity in different types of malignancies, including hepatocellular carcinoma, triple-negative breast cancer and multiple myeloma [13-16]. Several additional studies have examined the safety, tolerability and efficacy of tivantinib in combination with erlotinib [12, 17-20], gemcitabine [21], sorafenib [22] and irinotecan/cetuximab [23]. Two phase III studies of single agent tivantinib in patients with hepatocellular carcinoma are currently ongoing (NCT01755767, NCT02029157).

The mammalian target of rapamycin (mTOR) is a critical downstream component of the PI3K/AKT pathway. Temsirolimus (Torisel ${ }^{\mathrm{TM}}$, Pfizer Pharmaceuticals, Inc; Wyeth 
Pharmaceuticals Inc.) is a selective mTOR inhibitor and is approved for the treatment of renal cell carcinoma at a dose of $25 \mathrm{mg}$ intravenously weekly [24]. It is primarily metabolized by CYP3A4 and as such drugs that induce or inhibit CYP3A4/5 can affect its pharmacokinetics [25]. Based on the rationale that combined inhibition of c-Met and mTOR can lead to overcome of resistance and enhanced antitumor activity, we conducted a phase I trial of tivantinib in combination with temsirolimus in patients with advanced solid malignancies, followed by an expansion cohort. The primary objective of the study was to determine the MTD and the RP2D as well as to seek preliminary evidence of safety, tolerability and clinical activity of the above combination. Due to potential for pharmacokinetic (PK) interaction, PK analysis was also conducted.

\section{Material and methods}

\section{Patient selection}

Eligible patients were at least 18 years old with histologically confirmed advanced solid malignancy for which standard curative or palliative systemic therapies did not exist or were no longer effective. All patients were required to have measurable or evaluable disease, Eastern Cooperative Oncology Group performance status of 0-2, life expectancy of at least 12 weeks as well as adequate renal, liver and bone marrow reserve [defined as serum creatinine within institutional normal limit or creatinine clearance $\geq 60 \mathrm{~mL} / \mathrm{min} / 1.73 \mathrm{~m}^{2}$; aspartate transaminase/alanine transaminase $<2.5 \times$ upper limit of normal (ULN)and total bilirubin $<1.5 \times$ ULN that was later updated as per package insert (version 8/2014) to allow patients with mild hepatic impairment (bilirubin $>1-1.5 \times$ ULN or aspartate transaminase > ULN but bilirubin $\leq \mathrm{ULN}$ ) to receive temsirolimus at a reduced dose of $15 \mathrm{mg} / \mathrm{week}$; hemoglobin $\geq 9 \mathrm{~g} / \mathrm{dL}$, total white blood count $\geq 3.000 / \mu \mathrm{L}$, absolute neutrophil count $\geq$ $1.500 / \mu \mathrm{L}$ and platelet count $\geq 100.000 / \mu \mathrm{L}]$. In addition, patients were required to have normal phosphorus levels and a metabolic profile within normal limits (defined as fasting glucose $\leq 150 \mathrm{mg} / \mathrm{dL}$, fasting cholesterol $\leq 350 \mathrm{mg} / \mathrm{dL}$ and fasting triglycerides $\leq 300 \mathrm{mg}$ / dL). Patients with brain metastasis were allowed to enroll as long as their brain disease was treated and under control. Women of child-bearing potential were required to use adequate contraception.

Key exclusion criteria included recent treatment with chemotherapy, radiotherapy or endocrine therapy, incompletely resolved adverse events due to agents administered more than 4 weeks prior to study entry, previous anticancer therapy with mTOR or c-Met inhibitors, HIV infection, uncontrolled diabetes, clinically symptomatic hypothyroidism as well as history of allergic reactions attributed to compounds of similar chemical or biologic composition to tivantinib or temsirolimus. Concurrent use of CYP2C19 sensitive substrates, inducers or moderate/strong inhibitors as well as CYP3A4/5 inducers or moderate/strong inhibitors was prohibited.

The study was approved by the Institutional Review Board at the University of WisconsinMadison and was conducted in accordance to the Declaration of Helsinki for human subject protection. All patients provided written informed consent prior to enrollment. 


\section{Study design and treatment plan}

This is a single institution, open-label, phase I study. All patients were treated at the University of Wisconsin Carbone Cancer Center. Prior to enrollment, patients were screened for $C Y P 2 C 19 * 2$ and $* 3$ polymorphisms performed by a commercial vendor and were divided to extensive (cohort A) or poor metabolizers ( $* 2 / * 2, * 2 / * 3$ or $* 3 / * 3$; cohort $\mathrm{B}$ ), respectively. Dose escalation was planned separately for cohort A and cohort B. For cohort A, dose escalation was scheduled based on a traditional $3+3$ design, whereas for cohort $\mathrm{B}$ an accelerated dose escalation without intra-patient dose escalation was planned, due to the low prevalence of poor metabolizers. The primary objective of the study was to determine the MTD and the RP2D of tivantinib in combination with temsirolimus in patients with extensive (normal) CYP2C19 polymorphisms (cohort A). After the MTD was established for cohort A, 6 additional patients were planned to enroll in a dose expansion cohort at MTD (Figure 1).

In the dose escalation cohort, tivantinib was administered orally twice daily (BID) continuously and temsirolimus was administered intravenously weekly. Tivantinib was administered with food. Premedication with diphenhydramine 25-50 mg IV was administered approximately 30 minutes prior to initiation of temsirolimus infusion to prevent hypersensitivity reactions. Cycle 1 was 35 days, including a 7-day lead-in period with tivantinib alone, followed by infusion of the first dose of temsirolimus on day 8 . Subsequent cycles were 28 days. The starting dose of tivantinib and temsirolimus were 120 $\mathrm{mg}$ by mouth twice daily and $20 \mathrm{mg}$ intravenously weekly, respectively, and a stepwise dose escalation proceeded as per Table 1 . The only difference in the dose expansion cohort was that temsirolimus was started on day 1 of cycle 1 followed by the first dose of tivantinib on day 8.

\section{Safety and efficacy assessment}

History, physical examination, weight, ECOG performance status and standard laboratory analysis were obtained at baseline and prior to each cycle of treatment. Tumor assessment by either physical examination or imaging was performed at baseline and every two cycles of treatment thereafter. Other studies included EKG and fasting lipids at baseline and prior to each cycle. Pregnancy test for women of childbearing potential was also obtained at baseline.

Adverse events were assessed according to the National Cancer Institute Common Terminology Criteria for Adverse Events (CTCAE), version 4.0 (effective May 17, 2010). A DLT was defined as toxicity considered at least probably related to the study drugs that occurred between the first dose and the first day of cycle 2 and met the following criteria: grade $\geq 3$ nausea, vomiting, diarrhea, stomatitis, hyperlipidemia or rash uncontrolled despite maximal medical management; any other grade $\geq 3$ non-hematologic toxicity except grade 3 fatigue lasting < 7 days; inability to deliver more than $75 \%$ of the protocol specified cycle 1 treatment due to an at least probably treatment-related toxicity; dose delay of more than 7 days starting prior to day 1 of cycle 2 if the delay is due to an at least probably treatmentrelated toxicity; grade $\geq 3$ neutropenia lasting $>7$ days; grade 4 neutropenia or neutropenic fever; grade 3 thrombocytopenia lasting $>7$ days or of any duration if associated with a 
clinically significant or life-threatening bleed; grade 4 thrombocytopenia. Patients must have taken at least 75\% of ARQ 197 doses and at least 3 doses of temsirolimus to be considered evaluable. Any patient who did not complete the first scheduled cycle of therapy, but did not meet criteria for a DLT, was considered unevaluable and was replaced.

The MTD of the combination of tivantinib and temsirolimus was defined as the highest safely tolerated dose where less than $33 \%$ of patients experienced a DLT and two or more patients experienced a DLT at the next higher dose level. The RP2D was considered the MTD, unless the investigators determined a lower dose level based on post-cycle 1 observed adverse events or PK data.

All patients that received at least one dose of tivantinib or temsirolimus were evaluable for toxicity. Patients with measurable disease were evaluable for response if they received at least one cycle of therapy and had their disease re-evaluated. Response to treatment and progression were evaluated by the Response Evaluation Criteria in Solid Tumors (RECIST), version 1.1 [26]. Patients with lesions at baseline that did not meet the definitions of measurable disease were considered evaluable for non-target disease if they received at least 1 cycle of therapy and had their disease re-evaluated.

\section{Pharmacokinetic analysis}

Blood samples were collected prior to dosing and 0.5, 1, 2, 4, 8 and 12 hours after dosing for analysis of tivantinib on day 1 (first dose), 7 (tivantinib alone at steady state) and 8 (tivantinib at steady state and in combination with temsirolimus) of cycle 1 for patients in the dose escalation cohort. Samples were collected at the same timepoints on day 8 for analysis of temsirolimus. For patients in Arm 3, blood samples were collected prior to dosing and 0.5, 1, 2, 4, 8 and 12 hours after dosing for analysis of temsirolimus (temsirolimus alone first dose) on day 1 and day 8 of cycle 1 . Samples were collected at the same timepoints on day 8 for analysis of tivantinib (first dose). Tivantinib and temsirolimus plasma concentrations were analyzed by validated LC/MS/MS analyses as previously described [11,27]. Pharmacokinetic parameters were analyzed by noncompartmental methods using WinNonLin 6.4.

\section{Results}

\section{Patient characteristics}

Twenty-nine patients, 17 males and 12 females, with a median age of 58 years (range 28-77) were enrolled between July 2012 and July 2015. All patients were extensive (normal) CYP2C19 metabolizers. Baseline patient characteristics are summarized in Table 2.

\section{Dose escalation and toxicity}

DLTs by dose level are summarized in Table 3. Treatment was started with dose level 1 at $120 \mathrm{mg}$ BID of tivantinib and $20 \mathrm{mg}$ weekly of temsirolimus. Four patients were enrolled without experiencing DLT; one was unevaluable because she received $<75 \%$ of the study drugs due to progressive disease. Dose level 2 at $240 \mathrm{mg}$ BID of tivantinib and $20 \mathrm{mg}$ weekly of temsirolimus was then initiated. Of the first three patients, two experienced a 
DLT. One subject had grade 4 neutropenia and grade 3 mucositis probably related to therapy. The 2 nd patient was determined to be a DLT due to inability to deliver $>75 \%$ of cycle 1 drug therapy due to toxicities related to therapy. However, the highest grade toxicity was grade 3 hypophosphatemia which resolved rapidly with repletion and grade 3 diarrhea without optimal medical management due to lack of communication regarding symptoms. Diarrhea then resolved with medical management. Notably, this patient also had baseline grade 2 intermittent diarrhea from prior therapies for which atropine/diphenoxylate was started just prior to study entry.

Per protocol, dose level 1 was expanded. Four patients were added, three completed dose level 1 without DLT and one was unevaluable. After discussion with the sponsor, it was determined that since the second DLT at dose level two was potentially avoidable with more restrictive exclusion criteria for baseline diarrhea and more intensive management on study. Thus, the protocol was amended to expand enrollment at dose level 2. No additional DLTs were noted, thus, 1 of 6 evaluable patients at dose level 2 experienced a DLT.

Dose level 3 enrolled a total of 5 patients at $360 \mathrm{mg}$ BID of tivantinib and $20 \mathrm{mg}$ weekly of temsirolimus. One patient was unevaluable. Two patients experienced DLTs: one had grade 3 abdominal pain and the other grade 4 neutropenia/grade 3 febrile neutropenia. As such, the MTD was established at $240 \mathrm{mg}$ twice daily of tivantinib and $20 \mathrm{mg}$ weekly of temsirolimus. Eight additional patients were treated at dose level 2 in the expansion cohort. Only 1 out of 6 evaluable patients experienced a DLT, due to the inability to deliver $>75 \%$ of the protocol specified cycle 1 treatment due to grade 2 mucositis related to therapy.

The most common at least possibly related to treatment grade $\geq 2$ adverse events are summarized in Table 4. The most common were anemia, fatigue, anorexia and hypoalbuminemia. The most common grade 3 adverse events were hypophosphatemia, anemia, hypertension and hyponatremia. There were two patients with grade 4 neutropenia. No grade 5 adverse events were noted.

\section{Antitumor effect}

Twenty-seven patients were evaluable for response to therapy. Best clinical responses by dose level are listed in Table 5. Patients remained on study for a median of 71 days (range 18-296). A confirmed partial response (PR) was noted in one patient and stable disease (SD) in eight. The PR was seen in a heavily pretreated patient with ovarian cancer. Previous systemic treatments included carboplatin/paclitaxel, liposomal doxorubicin, topotecan, investigational drug huKS-IL2 and rechallenge with paclitaxel. She remained on study for a total of 296 days. Two patients had stable disease for prolonged period of time and remained on study for 206 (squamous cell carcinoma of the tongue) and 182 days (ovarian cancer), respectively.

\section{Pharmacokinetics}

To compare the pharmacokinetics of tivantinib after a single dose and at steady state, sampling was performed on Day 1 and Day 7 in the dose escalation cohort (Table 6). As expected, the dose adjusted Cmax and AUC after a single dose of tivantinib on Day 1 were significantly lower than on Day 7 when tivantinib was at steady state with geometric mean 
ratios of 0.80 (95\% CI: $0.78-0.82$ ) and 0.78 (95\% CI: 0.72-0.82) for the Cmax and AUC respectively.

Since both tivantinib and temsirolimus are substrates of CYP3A4 the potential for drug interactions with the combination was also assessed. To determine the effect of temsirolimus on tivantinib concentrations, tivantinib concentrations of Day 7, where tivantinib was at steady state and without temsirolimus and Day 8 when temsirolimus was administered are compared in the dose escalation cohort, with geometric mean ratios of 1.04 (95\% CI: 1.00 1.05) for the Cmax and 0.98 (95\% CI: 0.97-1.00) for the AUC and suggesting that temsirolimus does not alter tivantinib plasma concentrations.

To assess the influence of tivantinib on temsirolimus concentrations, individuals in the dose expansion cohort had a run-in with temsirolimus, where temsirolimus was administered on Day 1, and tivantinib was not started until Day 8. Pharmacokinetic parameters of temsirolimus from Day 1 in the dose expansion cohort were compared to the first dose of temsirolimus administered on Day 8 in the dose escalation cohort where tivantinib was at steady state showing a geometric mean ratio of 0.94 (95\% CI: 0.78-1.08) and 1.08 (95\% CI: 0.86-1.28), respectively for the Cmax and AUC and suggesting that tivantinib does not influence temsirolimus plasma concentrations.

\section{Discussion}

Dysregulated c-Met activity results in downstream activation of the PI3K/AKT/mTOR signaling pathway, with subsequent implications in the progression and spread of several malignancies $[3,4,28]$. This phase I study was designed to determine the MTD/RP2D of the combination of the c-Met inhibitor tivantinib with the mTOR inhibitor temsirolimus as well as to seek preliminary data of safety and efficacy in patients with advanced solid tumors. The rationale for combining these two agents is that dual inhibition of upstream c-Met and downstream mTOR may have a synergistic effect with enhanced anti-tumor activity compared to c-Met inhibition alone [29].

At the doses administered in this trial, the combination of tivantinib with temsirolimus was overall well tolerated. Most common grade $\geq 2$ adverse events were anemia, fatigue, anorexia, hypoalbuminemia, hypophosphatemia and nausea. The MTD/RP2D was determined to be $240 \mathrm{mg}$ orally twice daily for tivantinib in combination with $20 \mathrm{mg}$ intravenously weekly for temsirolimus. These doses were lower compared to the recommended single agent doses for both drugs; however, are doses at which clinical activity has been reported for each drug [11,24]. There was an interesting signal of antitumor activity from the combination of tivantinib and temsirolimus in diseases not typically thought to be responsive to mTOR inhibition. One patient with ovarian cancer was noted to have a PR and remained on the study for 10 months. In addition, 8 patients developed SD with 2 of them remaining on study for 6 and 7 months, respectively.

Tivantinib is primarily metabolized by CYP2C19 and partially by CYP3A4/5 [9]. Temsirolimus is a both a sensitive CYP3A4 substrate and a weak inhibitor of CYP3A4, with well-known drug interactions with CYP3A4 inducers and inhibitors and recommendations 
for dose modification with concurrent use [31]. Therefore, tivantinib and temsirolimus may both compete for metabolism via CYP3A4 and temsirolimus may inhibit the metabolism of tivantinib, representing a complex drug interaction scenario where pharmacokinetic studies are warranted. Our analysis demonstrated no interaction between temsirolimus and tivantinib which suggest these two CYP3A4 substrates can be safely co-administered. Whether this is generalizable to co-administration of other CYP3A4 substrates is unknown, although of considerable interest. In addition, it is well known that CYP2C19 is subject to genetic polymorphism that can alter metabolism of CYP2C19 substrates [30]. The most common polymorphisms that cause poor substrate metabolism are $C Y P 2 C 19 * 2$ and $* 3$, whereas CYP2C19*1 is considered the wild type [32]. Thus, we screened all patients prior to study entry and we planned for two separate cohorts, one for extensive (normal) and one for poor metabolizers. Due to the rarity of these poor metabolizer polymorphisms, all patients in our study were carriers of the wild type gene and as such were considered extensive (normal) metabolizers.

Previous studies have tested tivantinib in hepatocellular carcinoma as single agent [14] as well as in non-small cell lung cancer in combination with erlotinib [12, 17-20]. Although some success has been noted, there continue to be significant challenges. In patients with hepatocellular carcinoma, treatment with tivantinib was associated with a prolonged time to progression compared to placebo (1.6 vs. 1.4 months; HR 0.64, 90\% CI 0.43-0.94; $\mathrm{p}=0.04$ ), especially in patients with high expression of c-Met (2.7 vs. 1.4 months; HR 0.43, 95\% CI $0.19-0.97 ; p=0.03$ ) [14]. As such, two phase III studies in that setting are currently ongoing (NCT01755767, NCT02029157). In non-small cell lung cancer, the initial phase II study with tivantinib in combination with erlotinib showed promising results [17]; however, subsequent phase III studies either failed to confirm the superiority of the combination [20], or terminated before completing accrual due to increased incidence of interstitial lung disease [19]. One possible factor that could explain the lack of superiority of the combination is the mechanism of action of tivantinib itself. Even though tivantinib was initially designed as a c-Met inhibitor [8] with an initial phase I study providing evidence of pharmacodymanic effect of tivantinib on c-Met [10], subsequent in vitro studies have shown that tivantinib exerts its cytotoxic effect irrespective of c-Met inhibition [33, 34]. As such, tivantinib might not have an effect on c-Met amplification that is an established mechanism of resistance to EGFR TKIs like erlotinib.

In conclusion, the combination of tivantinib and temsirolimus was well tolerated and demonstrated promising anti-tumor activity. Based on our results, we believe that such a combination warrants further investigation.

\section{Acknowledgments}

The investigators gratefully acknowledge the patients and families who participated in this study. Funding was provided by the NCI Cancer Center Support Grant P30 CA014520 to University of Wisconsin Carbone Cancer Center and NCI U01CA062491-19S1 Early Clinical Trials of Anti-Cancer Agents with Phase I Emphasis.

\section{References}

1. Robinson DR, Wu YM, Lin SF. The protein tyrosine kinase family of the human genome. Oncogene. 2000; 19(49):5548-5557. [PubMed: 11114734] 
2. Zwick E, Bange J, Ullrich A. Receptor tyrosine kinase signalling as a target for cancer intervention strategies. Endocr Relat Cancer. 2001; 8(3):161-173. [PubMed: 11566607]

3. Birchmeier C, Birchmeier W, Gherardi E, Vande Woude GF. Met, metastasis, motility and more. Nat Rev Mol Cell Biol. 2003; 4(12):915-925. [PubMed: 14685170]

4. Gherardi E, Birchmeier W, Birchmeier C, Vande Woude G. Targeting MET in cancer: rationale and progress. Nat Rev Cancer. 2012; 12(2):89-103. [PubMed: 22270953]

5. Schmidt L, Duh FM, Chen F, et al. Germline and somatic mutations in the tyrosine kinase domain of the MET proto-oncogene in papillary renal carcinomas. Nat Genet. 1997; 16(1):68-73. [PubMed: 9140397]

6. Christensen JG, Schreck R, Burrows J, et al. A selective small molecule inhibitor of c-Met kinase inhibits c-Met-dependent phenotypes in vitro and exhibits cytoreductive antitumor activity in vivo. Cancer Res. 2003; 63(21):7345-7355. [PubMed: 14612533]

7. Furge KA, Zhang YW, Vande Woude GF. Met receptor tyrosine kinase: enhanced signaling through adapter proteins. Oncogene. 2000; 19(49):5582-5589. [PubMed: 11114738]

8. Munshi N, Jeay S, Li Y, et al. ARQ 197, a novel and selective inhibitor of the human c-Met receptor tyrosine kinase with antitumor activity. Mol Cancer Ther. 2010; 9(6):1544-1553. [PubMed: 20484018]

9. Investigator's Brochure. ARQ 197 (Version 5.0 O, 2010). Daiichi Sankyo Pharma Development; Edison, $\mathrm{NJ}$ :

10. Yap TA, Olmos D, Brunetto AT, et al. Phase I trial of a selective c-MET inhibitor ARQ 197 incorporating proof of mechanism pharmacodynamic studies. J Clin Oncol. 2011; 29(10):12711279. [PubMed: 21383285]

11. Rosen LS, Senzer N, Mekhail T, et al. A phase I dose-escalation study of Tivantinib (ARQ 197) in adult patients with metastatic solid tumors. Clin Cancer Res. 2011; 17(24):7754-7764. [PubMed: 21976535]

12. Yamamoto N, Murakami H, Hayashi H, et al. CYP2C19 genotype-based phase I studies of a c-Met inhibitor tivantinib in combination with erlotinib, in advanced/metastatic non-small cell lung cancer. Br J Cancer. 2013; 109(11):2803-2809. [PubMed: 24169346]

13. Wagner AJ, Goldberg JM, Dubois SG, et al. Tivantinib (ARQ 197), a selective inhibitor of MET, in patients with microphthalmia transcription factor-associated tumors: results of a multicenter phase 2 trial. Cancer. 2012; 118(23):5894-5902. [PubMed: 22605650]

14. Santoro A, Rimassa L, Borbath I, et al. Tivantinib for second-line treatment of advanced hepatocellular carcinoma: a randomised, placebo-controlled phase 2 study. Lancet Oncol. 2013; 14(1):55-63. [PubMed: 23182627]

15. Tolaney SM, Tan S, Guo H, et al. Phase II study of tivantinib (ARQ 197) in patients with metastatic triple-negative breast cancer. Invest New Drugs. 2015; 33(5):1108-1114. [PubMed: 26123926]

16. Dorkhom, SJ., et al. Phase II study of the c-Met inhibitor ARQ 197 (tivantinib) in patients with relapsed multiple myeloma. (https://ash.confex.com/ash/2012/webprogram/Paper53995.html)

17. Sequist LV, von Pawel J, Garmey EG, et al. Randomized phase II study of erlotinib plus tivantinib versus erlotinib plus placebo in previously treated non-small-cell lung cancer. J Clin Oncol. 2011; 29(24):3307-3315. [PubMed: 21768463]

18. Goldman JW, Laux I, Chai F, et al. Phase 1 dose-escalation trial evaluating the combination of the selective MET (mesenchymal-epithelial transition factor) inhibitor tivantinib (ARQ 197) plus erlotinib. Cancer. 2012; 118(23):5903-5911. [PubMed: 22605616]

19. Yoshioka H, Azuma K, Yamamoto N, et al. A randomized, double-blind, placebo-controlled, phase III trial of erlotinib with or without a c-Met inhibitor tivantinib (ARQ 197) in Asian patients with previously treated stage IIIB/IV nonsquamous nonsmall-cell lung cancer harboring wild-type epidermal growth factor receptor (ATTENTION study). Ann Oncol. 2015; 26(10):2066-2072. [PubMed: 26153496]

20. Scagliotti G, von Pawel J, Novello S, et al. Phase III Multinational, Randomized, Double-Blind, Placebo-Controlled Study of Tivantinib (ARQ 197) Plus Erlotinib Versus Erlotinib Alone in Previously Treated Patients With Locally Advanced or Metastatic Nonsquamous Non-Small-Cell Lung Cancer. J Clin Oncol. 2015; 33(24):2667-2674. [PubMed: 26169611] 
21. Pant S, Saleh M, Bendell J, et al. A phase I dose escalation study of oral c-MET inhibitor tivantinib (ARQ 197) in combination with gemcitabine in patients with solid tumors. Ann Oncol. 2014; 25(7):1416-1421. [PubMed: 24737778]

22. Puzanov I, Sosman J, Santoro A, et al. Phase 1 trial of tivantinib in combination with sorafenib in adult patients with advanced solid tumors. Invest New Drugs. 2015; 33(1):159-168. [PubMed: 25294187]

23. Eng C, Bessudo A, Hart LL, et al. A randomized, placebo-controlled, phase $1 / 2$ study of tivantinib (ARQ 197) in combination with irinotecan and cetuximab in patients with metastatic colorectal cancer with wild-type KRAS who have received first-line systemic therapy. Int J Cancer. 2016; 139(1):177-186. [PubMed: 26891420]

24. Hudes G, Carducci M, Tomczak P, et al. Temsirolimus, interferon alfa, or both for advanced renalcell carcinoma. N Engl J Med. 2007; 356(22):2271-2281. [PubMed: 17538086]

25. Boni JP, Hug B, Leister C, Sonnichsen D. Intravenous temsirolimus in cancer patients: clinical pharmacology and dosing considerations. Semin Oncol. 2009; 36(Suppl 3):S18-25. [PubMed: 19963096]

26. Eisenhauer EA, Therasse P, Bogaerts J, et al. New response evaluation criteria in solid tumours: revised RECIST guideline (version 1.1). Eur J Cancer. 2009; 45(2):228-247. [PubMed: 19097774]

27. Raymond E, Alexandre J, Faivre S, et al. Safety and pharmacokinetics of escalated doses of weekly intravenous infusion of CCI-779, a novel mTOR inhibitor, in patients with cancer. J Clin Oncol. 2004; 22(12):2336-2347. [PubMed: 15136596]

28. Garajová I, Giovannetti E, Biasco G, Peters GJ. c-Met as a Target for Personalized Therapy. Transl Oncogenomics. 2015; 7(Suppl 1):13-31. [PubMed: 26628860]

29. Ma PC, Schaefer E, Christensen JG, Salgia R. A selective small molecule c-MET Inhibitor, PHA665752, cooperates with rapamycin. Clin Cancer Res. 2005; 11(6):2312-2319. [PubMed: 15788682]

30. Chang SM, Kuhn J, Wen P, et al. Phase I/pharmacokinetic study of CCI-779 in patients with recurrent malignant glioma on enzyme-inducing antiepileptic drugs. Invest New Drugs. 2004; 22(4):427-435. [PubMed: 15292713]

31. Rodriguez-Antona C, Ingelman-Sundberg M. Cytochrome P450 pharmacogenetics and cancer. Oncogene. 2006; 25(11):1679-1691. [PubMed: 16550168]

32. Desta Z, Zhao X, Shin JG, Flockhart DA. Clinical significance of the cytochrome P450 2C19 genetic polymorphism. Clin Pharmacokinet. 2002; 41(12):913-958. [PubMed: 12222994]

33. Basilico C, Pennacchietti S, Vigna E, et al. Tivantinib (ARQ197) displays cytotoxic activity that is independent of its ability to bind MET. Clin Cancer Res. 2013; 19(9):2381-2392. [PubMed: 23532890]

34. Katayama R, Aoyama A, Yamori T, et al. Cytotoxic activity of tivantinib (ARQ 197) is not due solely to c-MET inhibition. Cancer Res. 2013; 73(10):3087-3096. [PubMed: 23598276] 


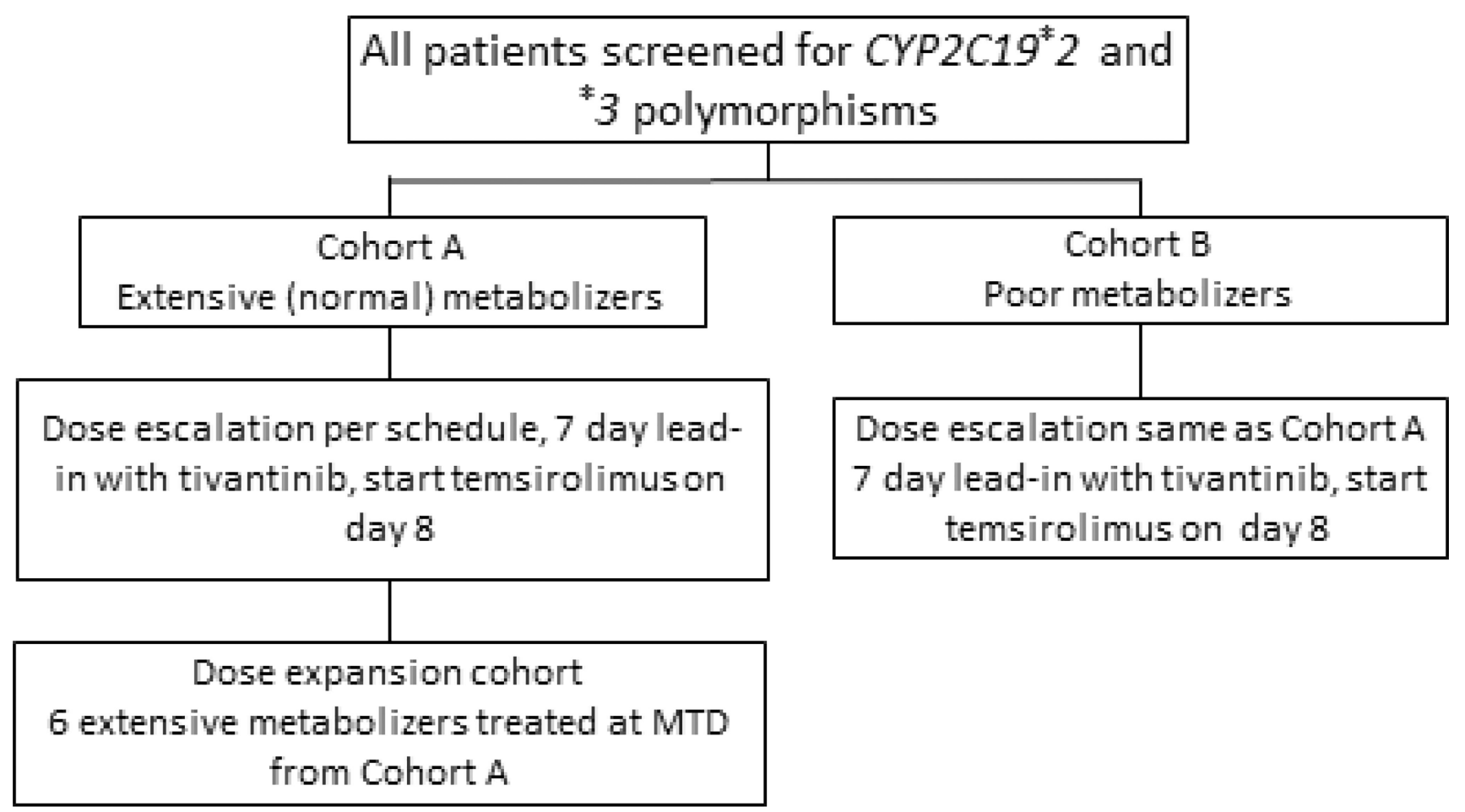

Figure 1.

Study design 
Table 1

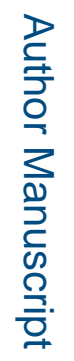

Dose escalation

\begin{tabular}{|c|c|c|}
\hline \multirow{2}{*}{ Dose level } & \multicolumn{2}{|c|}{ Dose } \\
\cline { 2 - 3 } & $\begin{array}{c}\text { Tivantinib } \\
\text { (mg orally twice daily) }\end{array}$ & $\begin{array}{c}\text { Temsirolimus } \\
\text { (mg intravenously weekly) }\end{array}$ \\
\hline Level -1 & 120 & 15 \\
\hline Level 1 & 120 & 20 \\
\hline Level 2 & 240 & 20 \\
\hline Level 3 & 360 & 20 \\
\hline Level 4 & 360 & 25 \\
\hline Level 5 & 480 & 25 \\
\hline
\end{tabular}




\section{Table 2}

Patient characteristics

\begin{tabular}{|c|c|c|}
\hline \multicolumn{2}{|l|}{ Characteristics } & $(\mathrm{N}=\mathbf{2 9})$ \\
\hline \multicolumn{2}{|c|}{ Age in years, median (range) } & $58(28-77)$ \\
\hline \multirow[t]{2}{*}{ Gender, $\mathbf{n}(\%)$} & Male & $17(59)$ \\
\hline & Female & $12(41)$ \\
\hline \multirow[t]{3}{*}{ ECOG PS, n (\%) } & 0 & $5(17)$ \\
\hline & 1 & $21(73)$ \\
\hline & 2 & $3(10)$ \\
\hline \multirow[t]{6}{*}{ Malignancy, n (\%) } & Colorectal & $9(31)$ \\
\hline & Ovarian & $6(21)$ \\
\hline & Non-small cell lung & $3(10)$ \\
\hline & Renal & $2(7)$ \\
\hline & Pancreatic & $2(7)$ \\
\hline & Cholangiocarcinoma, bladder, endometrial, tongue, esophageal, liposarcoma, carcinosarcoma & 1 each (24) \\
\hline \multirow[t]{2}{*}{ CYP2C19 status, n (\%) } & Extensive metabolizer & $29(100)$ \\
\hline & Poor metabolizer & $0(0)$ \\
\hline
\end{tabular}




\section{Table 3}

Dose levels and DLTs

\begin{tabular}{|c|c|c|c|}
\hline Dose Level & Enrolled & Unevaluable & DLT \\
\hline 1 & 8 & 2 & None \\
\hline 2 & 8 & 2 & $1^{*}$ \\
\hline 3 & 5 & 1 & $2^{* *}$ \\
\hline Expansion & 8 & 2 & $1^{* * *}$ \\
\hline
\end{tabular}
Grade 4 neutropenia and grade 3 mucositis,
*** One patient with grade 3 abdominal pain, one patient with grade 4 neutropenia/grade 3 febrile neutropenia,
*** Grade 2 mucositis that resulted to inability to deliver > $75 \%$ of the protocol specified cycle 1 treatment

2 mucositis that resulted to inability to deliver $>75 \%$ of the protocol specified cycle 1 treatment 
Table 4

At least possibly related to treatment grade $\geq 2$ adverse events experienced by at least 3 patients

\begin{tabular}{|l|c|c|c|c|}
\hline \multirow{2}{*}{ Adverse Event * } & \multicolumn{4}{|c|}{ Patients (N=29) } \\
\cline { 2 - 5 } & $\begin{array}{c}\text { Grade 2 } \\
\mathbf{n}(\boldsymbol{\%})\end{array}$ & $\begin{array}{c}\text { Grade 3 } \\
\mathbf{n}(\boldsymbol{\%})\end{array}$ & $\begin{array}{c}\text { Grade 4 } \\
\mathbf{n}(\boldsymbol{\%})\end{array}$ & $\begin{array}{c}\text { Overall } \\
\mathbf{n}(\boldsymbol{\%})\end{array}$ \\
\hline Anemia & $9(31)$ & $3(10.3)$ & 0 & $12(41.4)$ \\
\hline Fatigue & $10(34.5)$ & 0 & 0 & $10(34.5)$ \\
\hline Anorexia & $9(31)$ & 0 & 0 & $9(31)$ \\
\hline Hypoalbuminemia & $6(20.7)$ & $2(6.9)$ & 0 & $8(27.6)$ \\
\hline Hypophosphatemia & $2(6.9)$ & $5(17.2)$ & 0 & $7(24.1)$ \\
\hline Nausea & $6(20.7)$ & $1(3.4)$ & 0 & $7(24.1)$ \\
\hline Mucositis & $5(17.2)$ & $1(3.4)$ & 0 & $6(20.7)$ \\
\hline Vomiting & $3(10.3)$ & $2(6.9)$ & 0 & $5(17.2)$ \\
\hline Diarrhea & $4(13.8)$ & $1(3.4)$ & 0 & $5(17.2)$ \\
\hline Hypokalemia & $4(13.8)$ & $1(3.4)$ & 0 & $5(17.2)$ \\
\hline Hypertension & $2(6.9)$ & $3(10.3)$ & 0 & $5(17.2)$ \\
\hline Dyspnea & $2(6.9)$ & $2(6.9)$ & 0 & $4(13.8)$ \\
\hline Leukopenia & $2(6.9)$ & $1(3.4)$ & $1(3.4)$ & $4(13.8)$ \\
\hline Abdominal pain & $1(3.4)$ & $2(6.9)$ & 0 & $3(10.2)$ \\
\hline Dehydration & $2(6.9)$ & $1(3.4)$ & 0 & $3(10.2)$ \\
\hline Hyperglycemia & $2(6.9)$ & $1(3.4)$ & 0 & $3(10.2)$ \\
\hline Hyponatremia & 0 & $3(10.3)$ & 0 & $3(10.2)$ \\
\hline Neutropenia & $1(3.4)$ & 0 & $2(6.9)$ & $3(10.2)$ \\
\hline Thrombocytopenia & $3(10.3)$ & 0 & 0 & $3(10.2)$ \\
\hline
\end{tabular}

No Grade 5 Adverse Events were noted 


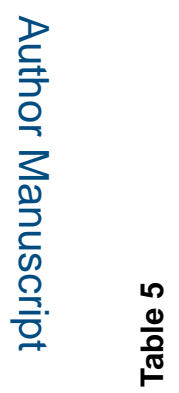

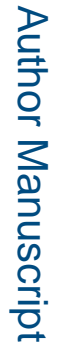

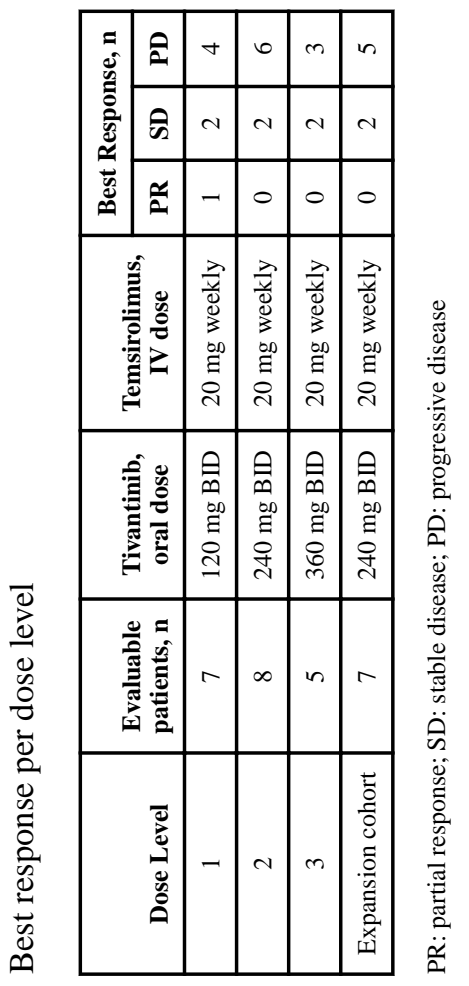

롤

Invest New Drugs. Author manuscript; available in PMC 2018 February 12. 


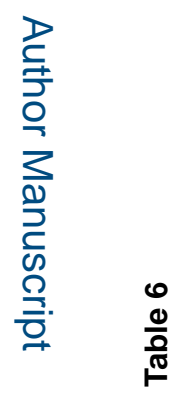

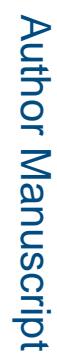

\begin{tabular}{|c|c|c|c|c|c|c|c|c|c|c|c|c|c|c|}
\hline & 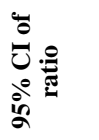 & $\begin{array}{l}0 \\
0 \\
0 \\
\infty \\
0 \\
0 \\
0\end{array}$ & & 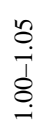 & & 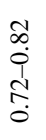 & & $\begin{array}{l}8 \\
\stackrel{8}{1} \\
\hat{a} \\
0\end{array}$ & & & $\mid \begin{array}{l}\infty \\
\stackrel{0}{0} \\
\dot{1} \\
\infty \\
0 \\
0\end{array}$ & & 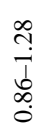 & \\
\hline$\frac{m}{b}$ & 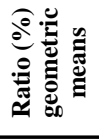 & $\begin{array}{l}0 \\
\infty \\
0\end{array}$ & & $\underset{\overbrace{}}{ \pm}$ & & $\stackrel{\substack{0 \\
0}}{0}$ & & $\begin{array}{l}\infty \\
\stackrel{0}{0}\end{array}$ & & & $\begin{array}{l} \pm \\
0 \\
0\end{array}$ & & $\stackrel{g}{\sigma}$ & \\
\hline 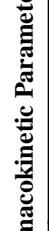 & 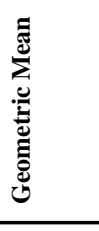 & 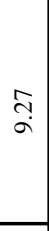 & $\begin{array}{l}\tilde{n} \\
= \\
=\end{array}$ & $\stackrel{n}{\stackrel{n}{=}}$ & $\stackrel{\overbrace{}}{=}$ & 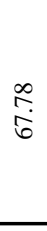 & $\begin{array}{l}\text { D. } \\
\infty \\
\infty\end{array}$ & $\begin{array}{l}0 \\
\infty \\
\infty \\
\infty\end{array}$ & $\begin{array}{l}t \\
\infty \\
\infty \\
\infty\end{array}$ & 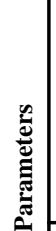 & $\mid \begin{array}{l}\tilde{r} \\
\text { id }\end{array}$ & $\begin{array}{l}\infty \\
\stackrel{\infty}{i} \\
\infty \\
\infty\end{array}$ & 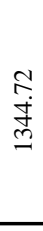 & 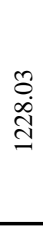 \\
\hline 意 & z & $\bar{\lambda}$ & $\vec{\sim}$ & $\bar{\lambda}$ & $\vec{\sim}$ & $\vec{\lambda}$ & $\bar{\lambda}$ & $\vec{\sim}$ & $\bar{\sim}$ & $\bar{\Xi}$ & $\infty$ & $\vec{\sim}$ & $\infty$ & $\bar{\lambda}$ \\
\hline 言 & 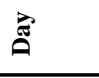 & - & - & $r$ & $\infty$ & - & r & $r$ & $\infty$ & 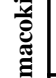 & - & $\infty$ & - & $\infty$ \\
\hline 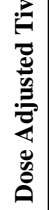 & 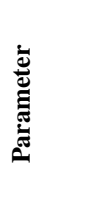 & 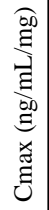 & & & & 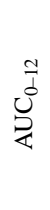 & 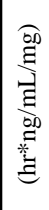 & & & 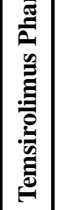 & 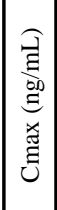 & & $\begin{array}{l}8 \\
\bigcup_{0}^{8} \\
己\end{array}$ & 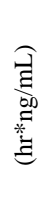 \\
\hline
\end{tabular}

